\title{
The impact of minimal extrathyroidal extension in the recurrence of papillary thyroid cancer patients
}

\author{
Maria Fernanda Ozorio de Almeida' \\ https://orcid.org/0000-0002-3451-7169 \\ Júlia Soares Couto' \\ https://orcid.org/0000-0001-8530-6962 \\ Ana Luiza Trevizani Ticly' \\ https://orcid.org/0000-0002-3979-5088 \\ Vivian Cenize Guardia' \\ https://orcid. org/0000-0002-1940-1686
}

Marilia Martins Silveira Marone ${ }^{2}$

https://orcid.org/0000-0002-5494-8281

Nilza Maria Scalissi ${ }^{1}$

https://orcid.org/0000-0003-2158-6613

Adriano Namo Cury ${ }^{1}$

https://orcid. org/0000-0002-9777-8366

Carolina Ferraz'

https://orcid.org/0000-0002-6620-8926

Rosália do Prado Padovani²

https://orcid.org/0000-0002-5497-6813

\begin{abstract}
Objective: We aimed to evaluate the impact of minimal extrathyroidal extension (mETE) alone on the risk of recurrence of papillary thyroid carcinoma (PTC). The impact of other factors, including multifocality, age, tumor size, and stimulated thyroglobulin (sTg) values was also assessed. Subjects and methods: We retrospectively analyzed 1,108 PTC patients from a medical institution, who presented tumors $\leq 4 \mathrm{~cm}$ without any adverse characteristics other than $\mathrm{mETE}$. Patients were classified according to their response to initial treatment 12 to 24 months after surgery as proposed by the 2015 American Thyroid Association (ATA) guideline. Statistical analysis was performed using multivariate logistic regression and receiver operating characteristic (ROC) curve. Results: In the multivariate logistic regression analysis, mETE did not have an impact on the response to initial treatment $(p=0.44)$, similar to multifocality, age, and tumor size. InitialTg value was the only variable associated with a poor response ( $p<0.01$, odds ratio $=1.303,95 \%$ confidence interval $1.25-1.36)$. The $\mathrm{ROC}$ analysis revealed that $\mathrm{Tg}$ was significant (area under curve $=0.8750$ ); the cutoff value of $\mathrm{sTg}$ as a predictor of poor response was $10 \mathrm{ng} / \mathrm{mL}$ (sensitivity $=72.2 \%$, specificity $=98.5 \%$ ). Conclusion: For low-risk PTC presenting mETE as the only aggressive feature, the initial sTg value is essential to identify patients who may have a poor response after initial treatment and benefit from further treatment. Arch Endocrinol Metab. 2020;64(3):251-6
\end{abstract}

Keywords

Differentiated thyroid cancer; minimal extrathyroidal extension; carcinoma extension; prognosis
1 Irmandade Santa da Casa de Misericórdia de São Paulo, São Paulo, SP, Brasil

${ }^{2}$ Irmandade da Santa Casa de Misericórdia de São Paulo, São Paulo, SP, Brasil; Departamento de Medicina Nuclear da Irmandade da Santa Casa de São Paulo, SP, Brasi

Correspondence to:

Rosália do Prado Padovani Irmandade Santa Casa de Misericórdia de São Paulo, Dr. Cesário Mota Júnior, 112, Vila Buarque

01221-020 - São Paulo, SP, Brasil rosaliapadovani@yahoo.com.br

Received on June/26/2019 Accepted on Jan/10/2020

DOI: 10.20945/2359-3997000000245

\section{INTRODUCTION}

$\mathrm{D}$ ifferentiated thyroid cancer (DTC) is the most common type of thyroid cancer, and it represents approximately $90 \%$ of all thyroid malignancies $(1,2)$; papillary thyroid carcinoma (PTC) is the most prevalent subtype of DTC. The prognosis of PTC patients is almost always favorable, and low-risk cases have an excellent prognosis with conservative treatment, with adequate surgery and TSH-suppressive thyroid hormone therapy $(1,2)$. Approximately $3 \%$ of these patients present tumor recurrence during decades of follow-up, and in general, only $1 \%-2 \%$ die of these thyroid tumors (1-3). Therefore, radioactive iodine remnant ablation is not routinely recommended after thyroidectomy for all American Thyroid Association (ATA) low-risk DTC patients (2). 
According to the ATA guideline (2), some pathological features are responsible for increasing the risk of persistence and recurrence of PTC, such as minimal extrathyroidal extension (mETE). mETE is characterized by tumor extension beyond the thyroid capsule to the perithyroid soft tissue or sternothyroid muscle alone (4), and it is observed in $5 \%-45 \%$ of all DTC patients $(2,5)$.

Considering the initial risk stratification proposed by the ATA, the presence of mETE alone upstages low-risk patients to the intermediate-risk group, independently of age $(2,6,7)$. It also suggests that the risk of recurrence related to $\mathrm{mETE}$ alone ranges from $3 \%-9 \%(6,7)$. Therefore, even when mETE is present without any other adverse features, more aggressive initial treatment is strongly recommended $(2,8,9)$.

The ATA (2), British Thyroid Association (10) and European Thyroid Association (8) are in favor of radioactive iodine therapy (RIT) when mETE is present, whereas French Societies (11) of Nuclear Medicine and Endocrinology only recommend RIT for tumors that exhibit mETE and are larger than $1 \mathrm{~cm}(11)$. In addition, some studies have shown that for patients with tumors exhibiting mETE, other factors such as tumor size (12-14) and the value of postoperative thyroglobulin ( $\mathrm{Tg}$ ) (12) should also be considered before making a decision regarding more aggressive treatment.

Although the $8^{\text {th }}$ edition of the American Joint Committee on Cancer/TNM staging system has minimized the impact of minimal extrathyroidal extension (mETE), down staging it from $\mathrm{T} 3$ to $\mathrm{Tl}$ classification [compared to the $7^{\text {th }}$ edition $(3)$ ] on the risk of death of papillary thyroid carcinoma (PTC) (15), some researchers have reported that mETE itself is related to a poor outcome (16-18), and major organizations $(2,19,9)$ endorse a more aggressive management when mETE is present. So, the real role of this feature as an isolated risk factor for recurrent or persistent disease has still been questioned.

Therefore, this study aimed to assess the clinical impact of mETE as a predictor of a worse response to initial treatment in PTC tumors. As a second endpoint, we evaluated the impact of other factors such as multifocality, tumor size, and initial stimulated $\mathrm{Tg}$ ( $\mathrm{s} T \mathrm{~g})$ value on the response to initial treatment.

\section{SUBJECTS AND METHODS}

We included PTC patients seen at the Nuclear Medicine Division at Santa Casa of São Paulo in this retrospective study. The study was approved by the local research ethics committee. The requirement for informed consent was waived, since there are no interventions on patients and most individuals no longer visit the institution. Low-risk patients and intermediate risk patients with tumors $\leq 4 \mathrm{~cm}$ were included. Tumors with others aggressive features as vascular invasion and aggressive histology and patients with clinically relevant lymph node metastases $(\mathrm{cNl})$ $(20,21)$ and positive antithyroglobulin antibodies were excluded. From the total database, 1,049 patients fulfilled the criteria to be included in the low-risk group and 59 patients in the mETE group. We included the low-risk group to evaluate the difference in risk and prognosis between the mETE group (intermediate-risk patients that receive this classification just because tumors present mETE) and the lowest risk patients corresponding the low risk stage. All patients underwent total thyroidectomy and hormone therapy with levothyroxine after surgery. No patients underwent RIT as an additional treatment. Elective lymph node dissection of the central compartment was not performed in any patient. All patients were reevaluated and reclassified 12 to 24 months after initial treatments according to the continuous risk stratification proposed by the 2015 ATA guideline $(2,5)$. Excellent and indeterminate responses were considered to indicate a good response, and incomplete response included patients with biochemical and structural disease. To perform this evaluation, we used non-stimulated $\mathrm{Tg}$ (non$\mathrm{s} \mathrm{Tg}$ ), stimulated $\mathrm{Tg}$ values ( $\mathrm{T} \mathrm{Tg}$ ) and whole-body scan (WBS) image mainly, and other images exams as ultrasound and computed tomography when available. Since we collected data from a nuclear medicine department, we did not have complementary images from all the patients.

Serum $\mathrm{Tg}$ levels were assessed at least three months after total thyroidectomy with thyroid-stimulating hormone $>30 \mathrm{uUI} / \mathrm{mL}$ using the immulite $\mathrm{Tg}$ assay (Roche Diagnosis $^{\mathrm{TM}}$, Mannheim, Germany). This is a sensitive two-site chemiluminescent immunoassay and the lower limit of detection was $0.2 \mathrm{ng} / \mathrm{mL}$. We also analyzed the impact of some tumors characteristics as multifocality, tumor size and $\mathrm{sTg}$ in the response to initial treatment.

Statistical analysis was performed using multivariate logistic regression and receiver operating characteristic curve (ROC curve). 


\section{RESULTS}

Table 1 lists the clinical features of the 1,108 PTC patients included in our study. Of them, 1,049 were low-risk patients, and 59 indeterminate risk patients that had tumors $\leq 4 \mathrm{~cm}$ without any aggressive feature other than mETE. Women corresponded to $90.8 \%$ of the group, $75.2 \%$ of the patients were below 55 years of age at diagnosis. Regarding tumor size, the majority (61.9\%) of tumors was $<1 \mathrm{~cm}, 24.1 \%$ were between 1.0 and $2.0 \mathrm{~cm}$, and $14.0 \%$ were between 2.1 and $4.0 \mathrm{~cm}$. The sTg measurement was available for 1,002 patients. Using the continuous risk stratification proposed by the ATA 2015 guideline (2), patients were reevaluated according to the response after 12 to 24 months from the initial treatment.

Table 1. Characteristics of patients with papillary thyroid carcinoma

\begin{tabular}{|c|c|c|}
\hline Patient characteristics & No & $\%$ \\
\hline \multicolumn{3}{|l|}{ Number: 1,108 patients } \\
\hline \multicolumn{3}{|l|}{ Age at diagnosis } \\
\hline$<55$ years & 883 & 75.2 \\
\hline$\geq 55$ years & 275 & 24.8 \\
\hline \multicolumn{3}{|l|}{ Size } \\
\hline$<1 \mathrm{~cm}$ & 686 & 61.9 \\
\hline $1-2 \mathrm{~cm}$ & 267 & 24.1 \\
\hline $2-4 \mathrm{~cm}$ & 155 & 14.0 \\
\hline \multicolumn{3}{|l|}{ Sex } \\
\hline Female & 1,006 & 90.8 \\
\hline Male & 102 & 9.2 \\
\hline \multicolumn{3}{|l|}{ Extrathyroidal extension } \\
\hline No & 1,049 & 94.7 \\
\hline Yes & 59 & 5.3 \\
\hline \multicolumn{3}{|c|}{ Postoperative sTg values (ng/dL) } \\
\hline$<1$ & 412 & 37.2 \\
\hline $1-10$ & 451 & 40.7 \\
\hline$>10$ & 139 & 12.5 \\
\hline Not stated & 106 & 9.6 \\
\hline \multicolumn{3}{|l|}{ Response to initial treatment } \\
\hline Excellent & 29 & 2.6 \\
\hline Biochemical incomplete & 152 & 13.7 \\
\hline Structural incomplete & 35 & 3.2 \\
\hline Indeterminate & 892 & 80.5 \\
\hline
\end{tabular}

sTg: stimulated thyroglobulin; RIT: radioactive iodine therapy.

Patients with indeterminate response had non-sTg values that were detectable but $<1 \mathrm{ng} / \mathrm{mL}$ and $\mathrm{sTg}$ values between 1 and $10 \mathrm{ng} / \mathrm{mL}$ with no suspicious image.

We observed that $2.6 \%$ showed excellent response, $80.5 \%$ indeterminate response, $13.7 \%$ incomplete biochemistry and $3.2 \%$ incomplete structural response.

In the multivariate logistic regression analysis, mETE did not have an impact on the response to initial treatment $(\mathrm{p}=0.44)$, similar to multifocality $(\mathrm{p}=0.809)$, age $(\mathrm{p}=0.295)$, and tumor size $(\mathrm{p}=$ $0.385)$. Initial $\mathrm{Tg}$ value was the only variable associated with a poor response $(\mathrm{p}<0.01$, odds ratio $=1.303$, 95\% confidence interval 1.252-1.36) (Table 2). The ROC curve analysis revealed that $\mathrm{sTg}$ was significant (area under the curve $=0.8750$ ), and the cutoff value of $\mathrm{s} T \mathrm{~g}$ as a predictor of poor response was $10 \mathrm{ng} / \mathrm{mL}$ $($ sensitivity $=72.2 \%$ and specificity $=98.5 \%$; Figure 1$)$.

Table 2. Results of multivariate logistic regression analysis

\begin{tabular}{lc}
\hline & Multivariate logistic regression analysis \\
\hline Variables & p \\
\hline Age & 0.295 \\
Tumor size & 0.385 \\
Multifocality & 0.809 \\
mETE & 0.444 \\
Initial sTg & 0.000 \\
\hline
\end{tabular}

mETE: minimal extrathyroidal invasion; sTg: stimulated thyroglobulin.

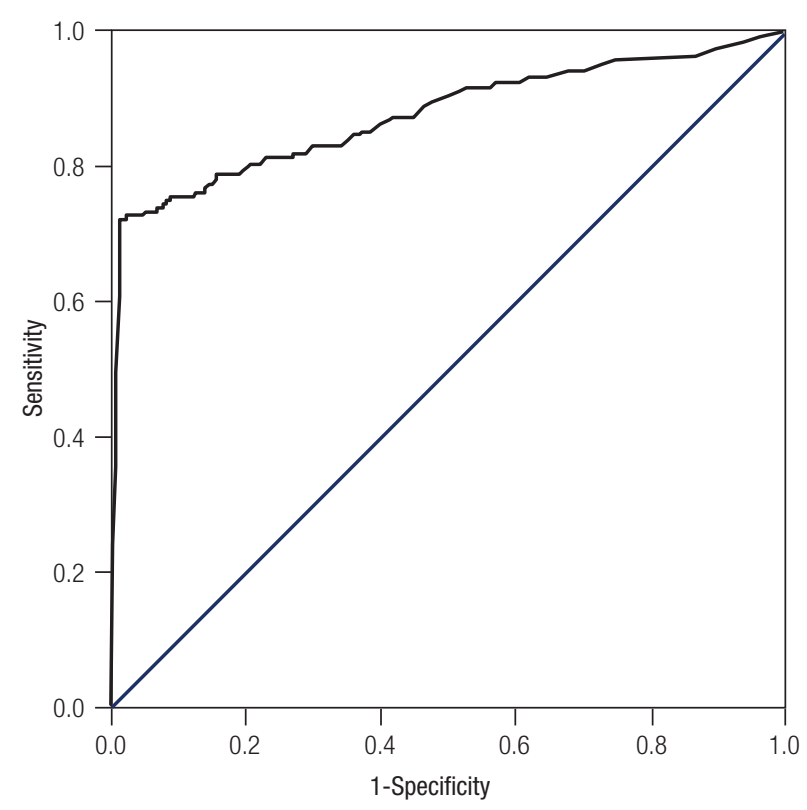

Figure 1. Receiver operating characteristic curve of stimulated thyroglobulin value.

\section{DISCUSSION}

Our study evaluated the impact of some tumor characteristics as the presence of $\mathrm{mETE}$, multifocality, tumor size, and initial sTg value in the response to the initial treatment of papillary thyroid cancer patients treated with surgery and not submited to RIT. sTg was 
the only variable that correlated with a worse response to the initial treatment excluding the impact of mETE and suggesting that the presence of this feature alone does not necessarily increase the risk of recurrence in DTC patients.

The recognition of $\mathrm{mETE}$ as an isolated risk factor of poor prognosis was questioned for the first time by Ito and cols. at Kuma Hospital (22). They demonstrated the lack of prognostic value of mETE alone and suggested that upgrading the category may not be appropriate (22). Following the same reasoning, the latest American Joint Committee on Cancer edition released in 2017 changed the stratification criteria and no longer considers mETE alone as an isolated risk factor for mortality (15). However, the impact of mETE on the recurrence risk remains controversial, and according to the 2015 ATA guideline (2), it should still be considered as an isolated risk factor.

Some studies have shown that mETE without concomitant gross extrathyroidal extension did not increase the rate of recurrence, similar to microscopic intrathyroidal tumors (23-27). However, unlike the previously cited studies, some researchers have reported contradictory results regarding the impact of mETE. A recent meta-analysis found that the presence of mETE is a factor for recurrence and decreased diseasefree survival (16), supporting the indication of more aggressive management for these patients. However, the quality of some studies included in this systematic review was not much satisfactory, according to the Newcastle-Ottawa Scale score. In contrast, our results showed that mETE alone was not associated with a worse response to initial treatment suggesting that the presence of this feature alone does not necessarily increase the risk of recurrence in DTC patients.

A highlight of this study is that we analyzed a subgroup of patients with mETE and no other known aggressive feature. Furthermore, all patients analyzed, independently of sTg value, did not undergo additional treatment after surgery. This is especially important when we consider the controversy of RIT necessity in patients that presented positive sTg after surgery. The 2015 ATA guideline states that RIT treatment is generally favored for patients with T3 tumors demonstrating mETE, considered as "ATA low-tointermediate risk" (2). Chow and cols. (28) described a cohort of 352 patients with mETE and demonstrated that RIT provided a good local control rate. In contrast, a systematic review by Lamartina and cols.
(29) concluded that mETE alone is not a sufficient justification for RIT because no difference was found in the rates of disease recurrence between patients with and without RIT. We demonstrated although mETE was not considered as an isolated risk factor for a worst prognosis, patients with tumors presenting $\mathrm{mETE}$ and $\mathrm{sTg}<10 \mathrm{ng} / \mathrm{mL}$ had a significant chance to respond better to initial treatment even without undergoing RIT. Moreover, patients whose tumors presented mETE and who had initial sTg > $10 \mathrm{ng} / \mathrm{mL}$ had a worst response and so, are likely to benefit from a more aggressive approach such as RIT since this group has a higher rate of incomplete response.

Concerning to the tumor size, many studies have evaluated the role of this characteristic, (specially in microcarcinomas), on the prognosis of PTC tumors (12-14,27,30,31); however, few studies found an association between tumor size and the presence of mETE (12-14). Buffet and cols. demonstrated that microcarcinomas presenting with mETE at diagnosis may benefit from RIT (30), and Rosario and cols. showed that mETE is related to a poor prognosis only if the tumor size is above $1.5 \mathrm{~cm}$ (12). We did not find any correlation between tumor size and a worst prognosis in the mETE group.

Zhi and cols. (14) showed that, in univariate and multivariate analysis, tumor size and also male gender and multifocality was significantly associated with lymph node metastasis and higher recurrence risk deserving cautious selection in surgery extent. Considering that our population has low risk features for prognosis, we could justify why we did not find any correlation between multifocality and tumor size with worst response to initial treatment, probably, in the higher risk group this data could not be extrapolated.

This study has some limitations. First, this was a retrospective analysis using data of patients who were not followed at the same center. Second, the number of patients with tumors presenting mETE was small. This is because the majority of patients with tumors presenting mETE are directed to our service to undergo RIT. Finally, an important observation is that the vast majority of patients have an indeterminate response and were not treated with radioiodine, more follow-up time and the thyroglobulin trend observation would allow stronger conclusions regarding the prognosis of this subgroup of patients. But, unfortunately, considering the data were collected from a nuclear medicine department, this information was not available for many patients. 
In conclusion, this study showed that mETE alone is not associated with a worse response to initial treatment; therefore, it should not be considered as an independent risk factor for recurrent disease during the management of DTC patients. Moreover, the presence of $\mathrm{mETE}$ should not independently influence the decision to perform RIT. Furthermore, initial sTg after surgery should be considered in patients with tumors presenting mETE as the only aggressive feature, and additional treatment should be recommended when $\mathrm{sTg}>10$ $\mathrm{ng} / \mathrm{mL}$. If $\mathrm{sTg}$ is $<10 \mathrm{ng} / \mathrm{mL}$, patients should benefit from follow-up without the need for a more aggressive approach. Our findings support the importance of individualized management of PTC patients.

Funding: this research did not receive any specific grant from any funding agency in the public, commercial or not-for-profit sector.

Disclosure: no potential conflict of interest relevant to this article was reported.

\section{REFERENCES}

1. National Cancer Institute. Surveillance, Epidemiology, and End Results (SEER). SEER Stat Fact Sheets: Thyroid Cancer [Internet]. 2016 [cited 2016 Jun 16] Available from: http://seer.cancer.gov/ statfacts/html/thyro.html.

2. Haugen BR, Alexander EK, Bible KC, Doherty GM, Mandel SJ, Nikiforov YE, et al. 2015 American Thyroid Association management guidelines for adult patients with thyroid nodules and differentiated thyroid cancer: The American Thyroid Association Guidelines Task Force on Thyroid Nodules and Differentiated Thyroid Cancer. Thyroid. 2016;26(1):1-133.

3. Yin DT, Yu K, Lu RQ, Li X, Xu J, Lei M. Prognostic impact of minimal extrathyroidal extension in papillary thyroid carcinoma. Medicine (Baltimore). 2016;95(52):e5794.

4. Mete O, Rotstein L, Asa SL. Controversies in thyroid pathology: thyroid capsule invasion and extrathyroidal extension. Ann Surg Oncol. 2010;17(2):386-91.

5. Tuttle RM, Tala H, Shah J, Leboeuf R, Ghossein R, Gonen M, et al. Estimating risk of recurrence in differentiated thyroid cancer after total thyroidectomy and radioactive iodine remnant ablation: using response to therapy variables to modify the initial risk estimates predicted by the new American Thyroid Association staging system. Thyroid. 2010;20(12):1341-9.

6. Riemann B, Krämer JA, Schmid KW, Dralle H, Dietlein M, Schicha $\mathrm{H}$, et al. Risk stratification of patients with locally aggressive differentiated thyroid cancer. Results of the MSDS trial. Nuklearmedizin. 2010;49(3):79-84.

7. Radowsky JS, Howard RS, Burch HB, Stojadinovic A. Impact of degree of extrathyroidal extension of disease on papillary thyroid cancer outcome. Thyroid. 2014;24(2):241-4.

8. Pacini F, Schlumberger M, Dralle H, Elisei R, Smit JW, Wiersinga $W$, et al. European consensus for the management of patients with differentiated thyroid carcinoma of the follicular epithelium. Eur J Endocrinol. 2006;154(6):787-803.

9. Rosário PW, Ward LS, Carvalho GA, Graf H, Maciel RMB, Maciel $L M Z$, et al. Thyroid nodules and differentiated thyroid cancer: update on the Brazilian consensus. Arq Bras Endocrinol Metab. 2013;57(4):240-64.

10. Perros P, Boelaert K, Colley S, Evans C, Evans RM, Gerrard BA G, et al.; British Thyroid Association. Guidelines for the management of thyroid cancer. Clin Endocrinol. 2014;81(1):1-122.

11. Zerdoud S, Giraudet AL, Leboulleux S, Leenhardt L, Bardet S, Clerc J, et al. Radioactive iodine therapy, molecular imaging and serum biomarkers for differentiated thyroid cancer: 2017 guidelines of the French Societies of Nuclear Medicine, Endocrinology, Pathology, Biology, Endocrine Surgery and Head and Neck Surgery. Ann Endocrinol. 2017;78(3):162-75.

12. Rosario PW, Mourão G, Calsolari1 MR. Risk of recurrence in patients with papillary thyroid carcinoma and minimal extrathyroidal extension not treated with radioiodine. $J$ Endocrinol Invest. 2019;42(6):687-92.

13. Ito $Y$, Fukushima M, Kihara M, Takamura $Y$, Kobayashi K, Miya A, et al. Investigation of the prognosis of patients with papillary thyroid carcinoma by tumor size. Endocr J. 2012;59(6):457-64.

14. Zhi J, Zhao J, Gao M, Pan Y, Wu J, Li Y, et al. Impact of major different variants of papillary thyroid microcarcinoma on the clinicopathological characteristics: the study of 1041 cases. Int J Clin Oncol. 2017;23(1):59-65.

15. Kim M, Kim WG, Oh HS, Park S, Kwon H, Song DE, et al. Comparison of the Seventh and Eighth Editions of the American Joint Committee on Cancer/Union for International Cancer ControlTumor-Node-Metastasis Staging System for Differentiated Thyroid Cancer. Thyroid. 2017;27(9):1149-55.

16. Edge SB, Compton CC. The American Joint Committee on Cancer: the 7th edition of the AJCC cancer staging manual and the future ofTNM. Ann Surg Oncol. 2010;17(6):1471-4.

17. Schindler AM, van Melle G, Evequoz B, Scazziga B. Prognostic factors in papillary carcinoma of the thyroid. Cancer. 1991;68(2):324-30.

18. Li JD, Chao TC, Weng HF, HoYS. Prognostic variables of papillary thyroid carcinomas with local invasion. Endocr J. 1999;46(1):91-8.

19. Leenhardt L, Erdogan MF, Hegedus L, Mandel SJ, Paschke R, Rago T, et al. 2013 European thyroid association guidelines for cervical ultrasound scan and ultrasound-guided techniques in the postoperative management of patients with thyroid cancer. EurThyroid J. 2013;2(3):147-59.

20. Sapuppo G, Palermo F, Russo M, Tavarelli M, Masucci R, Squatrito $\mathrm{S}$, et al. Latero-cervical lymph node metastases (N1b) represent an additional risk factor for papillary thyroid cancer outcome. J Endocrinol Invest. 2017;40(12):1355-63.

21. Park S, Kim WG, Song E, Oh HS, Kim M, Kwon H, et al. Dynamic risk stratification for predicting recurrence in patients with differentiated thyroid cancer treated without radioactive iodine remnant ablation therapy. Thyroid. 2017;27(4):524-30.

22. Ito $Y$, Tomoda C, Uruno T, Takamura Y, Miya A, Kobayashi K, et al. Prognostic significance of extrathyroid extension of papillary thyroid carcinoma: massive but not minimal extension affects the relapse-free survival. World J Surg. 2006;30(5):780-6.

23. Moon HJ, Kim EK, Chung WY, Yoon JH, Kwak JY. Minimal extrathyroidal extension in patients with papillary thyroid microcarcinoma: is it a real prognostic factor? Ann Surg Oncol. 2011;18(7):1916-23.

24. Al-Qurayshi Z, Shama MA, Randolph GW, Kandil E. Minimal extrathyroidal extension does not affect survival of welldifferentiated thyroid cancer. Endocr Relat Cancer. 2017;24(5):221-6.

25. Shin JH, Ha TK, Park HK, Ahn MS, Kim KH, Bae KB, et al. Implication of minimal extrathyroidal extension as a prognostic factor in papillary thyroid carcinoma. Int J Surg. 2013;11(9):944-7.

26. Nixon IJ, Ganly I, Patel S, Palmer FL, Whitcher MM, Tuttle RM, et al. The impact of microscopic extrathyroid extension on outcome 
in patients with clinical T1 and T2 well-differentiated thyroid cancer. Surgery. 2011;150(6):1242-9.

27. Tam S, Amit M, Boonsripitayanon M, Busaidy NL, Cabanillas $\mathrm{ME}$, Waguespack SG, et al. Effect of tumor size and minimal extrathyroidal extension in patients with differentiated thyroid cancer. Thyroid. 2018;28(8):982-90.

28. Chow SM, Yau S, Kwan CK, Poon PC, Law SC. Local and regional control in patients with papillary thyroid carcinoma: specific indications of external radiotherapy and radioactive iodine according to T and N categories in AJCC 6th edition. Endocr Relat Cancer. 2006;13(4):1159-72.
29. Lamartina L, Durante C, Filetti S, Cooper DS. Low-risk differentiated thyroid cancer and radioiodine remnant ablation: a systematic review of the literature. J Clin Endocrinol Metab. 2015;100(5):1748-61.

30. Buffet C, Golmard JL, Hoang C, Trésallet C, Du Pasquier Fédiaevsky $\mathrm{L}$, Fierrard $\mathrm{H}$, et al. Scoring system for predicting recurrences in patients with papillary thyroid microcarcinoma. Eur J Endocrinol. 2012;167(2):267-75.

31. Ito $Y$, Miyauchi A, Oda H, Kobayashi K, Kihara M, Miya A. Revisiting low-risk thyroid papillary microcarcinomas resected without observation: was immediate surgery necessary? World J Surg. 2016;40(3):523-8. 